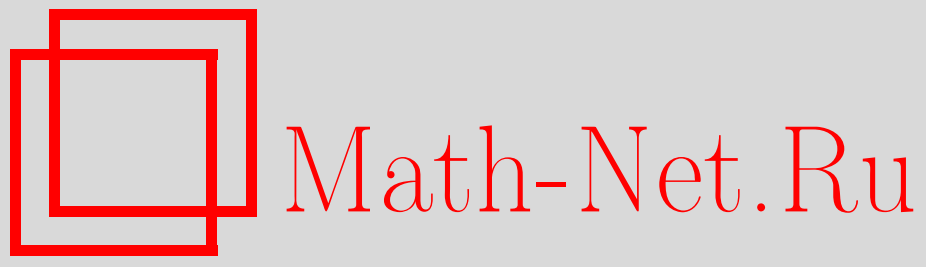

Г. А. Калябин, Точные константы в неравенствах для промежуточных производных (случай Габушина), Функи. анализ и его прил., 2004, том 38, выпуск $3,29-38$

DOI: https://doi.org/10.4213/faa115

Использование Общероссийского математического портала MathNet.Ru подразумевает, что вы прочитали и согласны с пользовательским соглашением

http://www . mathnet.ru/rus/agreement

Параметры загрузки:

IP : 54.197 .130 .99

26 апреля 2023 г., 07:46:29

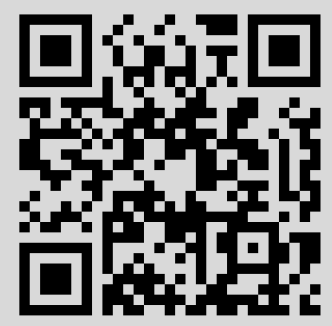


Функииональный анализ и его приложения

2004, т. 38 , вып. 3, с. 29-38

УДК 517.518.26

\title{
Точные константы в неравенствах для промежуточных производных (случай Габушина)*
}

\author{
(c) 2004. Г. А. КАляБин
}

Дорогому учителю в знак глубокого уважения и неизменной благодарности

\section{§1. Обозначения и формулировка основного результата}

Будем рассматривать семейство соболевских пространств $W_{2}^{n}\left(\mathbb{R}_{+}^{1}\right), n \in \mathbb{N}$, каждое из которых состоит из всех (комплекснозначных) функций $f(x)$, определенных на положительной полуоси $x \geqslant 0$, имеющих абсолютно непрерывную на любом отрезке $[0, b], b>0$, производную $f^{(n-1)}(x)$ порядка $n-1$ и обладающих конечной нормой

$$
\left\|f \mid W_{2}^{n}\left(\mathbb{R}_{+}^{1}\right)\right\|:=\left(\int_{0}^{+\infty}\left(|f(x)|^{2}+\left|f^{(n)}(x)\right|^{2}\right) d x\right)^{1 / 2} .
$$

Нашей целью является вычисление точных, т. е. наименьших возможных, констант в неравенствах типа Колмогорова

$$
\left|f^{(k)}(0)\right| \leqslant A_{n, k}\left\|f \mid W_{2}^{n}\left(\mathbb{R}_{+}^{1}\right)\right\|, \quad k \in I_{n}:=\{0, \ldots, n-1\} .
$$

Теорема. Для любого $k \in I_{n}$ наименъшая константа $A_{n, k}$ в (1.2) выражается формулой

$$
A_{n, k}=\left(\sin \frac{\pi(2 k+1)}{2 n}\right)^{-1 / 2} \prod_{s=1}^{k} \operatorname{ctg} \frac{\pi s}{2 n} ;
$$

в частности, при $k=0$ имеем

$$
A_{n, 0}=\left(\sin \frac{\pi}{2 n}\right)^{-1 / 2}
$$

Известные результаты о константах в неравенствах для промежуточных производных в различных случаях приведены, например, в $[1, \S 2.4]$. В частности, В. Н. Габушин [2] (см. также [1, п. 2.4.5]) нашел функции (в виде линейных комбинаций убывающих экспонент), экстремальные для неравенств (1.2). Однако неявно определяемые этим результатом числа $A_{n, k}$ не были вычислены эффективно. Например, оставалось неясным, как ведут себя при $n \rightarrow \infty$ числа $A_{n}:=\max _{k} A_{n, k}$.

Л. В. Тайков [3] (см. также [1, п. 2.4.4]) получил простую явную формулу для чисел $A_{n, k}^{\star}$, отличающихся от $A_{n, k}$ заменой в $(1.2)$ пространства $W_{2}^{n}\left(\mathbb{R}_{+}^{1}\right)$ функций, заданных на полуоси $\mathbb{R}_{+}^{1}$, пространством $W_{2}^{n}\left(\mathbb{R}^{1}\right)$ функций, заданных на

\footnotetext{
* Работа была поддержана грантами РФФИ (№02-01-00602) и CRDF (проект SA-014-02).
} 
всей оси $\mathbb{R}^{1}$, а именно, $A_{n, k}^{\star}=(2 n \sin ((2 k+1) \pi / 2 n))^{-1 / 2}$. Поэтому формула (1.3) может рассматриваться как обобщение результата Л. В. Тайкова на технически более сложный случай соболевских пространств на полуоси.

Доказательство теоремы излагается в 2 . В 33 в качестве следствий выводятся свойства симметрии и регулярности, дана таблица значений $A_{n, k}, n \leqslant 6$, выражающихся в радикалах, а в $\S 4$ изучается асимптотическое поведение констант $A_{n, k}$ при $n \rightarrow \infty$ для различных случаев поведения $k$.

Приведены также некоторые вспомогательные утверждения, которые могут представлять и самостоятельный интерес. Часть из них (предложения) были получены ранее [4], тогда как другие (леммъ) доказываются в настоящей работе, краткое содержание которой анонсировано в [8].

\section{§2. Доказательство теоремы}

Шаг 1. Предварительные рассмотрения. Для произвольного вектора $a:=$ $\left(a_{0}, a_{1}, \ldots, a_{n-1}\right)$ положим

$$
\psi_{n}(a):=\min \left\{\left\|f \mid W_{2}^{n}\left(\mathbb{R}_{+}^{1}\right)\right\|: y^{(s)}(0)=a_{s}, s \in I_{n}\right\} .
$$

Ясно, что $\psi_{n}^{2}(a)$ есть положительно определенная квадратичная форма на $n$-мерном пространстве векторов начальных условий $a, \psi_{n}^{2}(a)=\left(G_{n} a, a\right)$. Матрица $G=G_{n}$ подробно исследована в [4], где, в частности, установлены следующие ее свойства (см. $[4, \S 3])$.

ПрЕДЛОЖЕНИЕ 1. Матрища $G$ вещественна, симметрична относительно главной и побочной диагоналей и удовлетворяет тождеству

$$
G^{-1}=P G P, \quad P:=\operatorname{diag}\left(1,-1, \ldots,(-1)^{n-1}\right) .
$$

В частности, для нас большое значение имеет тот факт, вытекающий из важного и неожиданного соотношения (2.2), что диагональные элементы матрии, $G$ u $G^{-1}$ совпадают.

ЛЕмма 1. Для наименъших констант $A_{n, k}$ в неравенстве (1.2) справедливо альтернативное представление

$$
A_{n, k}=\min \left\{\left\|f \mid W_{2}^{n}\left(\mathbb{R}_{+}^{1}\right)\right\|: f^{(k)}(0)=1, f^{(j)}(0)=0, j \neq k, j \in I_{n}\right\} .
$$

ДокАЗАтЕЛЬСтво. Непосредственно из определяющего соотношения (1.2) вытекает, что $A_{n, k}$ суть числа, обратные к минимальным нормам в $W_{2}^{n}\left(\mathbb{R}_{+}^{1}\right)$ экстраполяций с заданным значением $f^{(k)}(0)=1$ при отсутствии условий на граничные значения других производных, т. е.

$$
A_{n, k}^{-1}=\min \left\{\left\|f \mid W_{2}^{n}\left(\mathbb{R}_{+}^{1}\right)\right\|: f^{(k)}(0)=1, f^{(j)}(0) \text { произвольны, } j \neq k, j \in I_{n}\right\} .
$$

Воспользуемся теперь тем, что для произвольной положительно определенной эрмитовой матрицы $Q$ и любого ненулевого вектора $b$ имеет место равенство

$$
\min \{(Q a, a):(a, b)=1\}=\left(Q^{-1} b, b\right)^{-1},
$$

являющееся простым (и хорошо известным) следствием неравенства КошиБуняковского. Выбирая в (2.4) $Q=G_{n}, b=e_{k}:=\left(\delta_{0, k}, \ldots, \delta_{n-1, k}\right)$, где, как обычно, $\delta_{j, k}$ равно 1 при $j=k$ и 0 при $j \neq k, k, j \in I_{n}$, и принимая во внимание, что условие $a_{k}=1$ при произвольности остальных $a_{s}$ в точности равносильно 
равенству $\left(a, e_{k}\right)=1$, мы заключаем, с учетом определения (см. $\left.(2.1)\right)$, что числа $A_{n, k}^{2}$ равны диагональным элементам матрицы, обратной к $G$, а поскольку диагонали матриц $G^{-1}$ и $G$ совпадают, мы приходим к $(2.3)$.

Шаг 2. Переход $к$ задаче на всей оси. Пусть $y_{k}(x), x>0$, - функция, удовлетворяющая начальным условиям, указанным в $(2.3)$, и имеющая наименьшую возможную (т. е. равную $\left.A_{n, k}\right)$ норму в $W_{2}^{n}\left(\mathbb{R}_{+}^{1}\right)$, и пусть $z_{k}(x), x \in \mathbb{R}^{1}$, обозначает четное (при четных $k$ ) или нечетное (при нечетных $k$ ) продолжение функции $y_{k}(x)$ на всю ось. Поскольку в точке $x=0$ имеет место непрерывность производных $z_{k}^{(s)}(x)$ до порядка $n-1$ включительно, продолженная функция принадлежит пространству Соболева на всей оси, причем (см (1.1)) $\left\|z_{k}\left|W_{2}^{n}\left(\mathbb{R}^{1}\right)\|=\sqrt{2}\| y_{k}\right| W_{2}^{n}\left(\mathbb{R}_{+}^{1}\right)\right\|=\sqrt{2} A_{n, k}$.

Используем теперь преобразование Фурье $F$ и обратное к нему $F^{-1}$ (см. $[1$, п. 1.6.2])

$$
F[f](\xi)=\hat{f}(\xi):=\frac{1}{2 \pi} \int_{-\infty}^{\infty} f(t) e^{-i \xi t} d t, \quad f(x)=F^{-1}[\hat{f}]=\int_{-\infty}^{\infty} \hat{f}(\xi) e^{i \xi x} d \xi
$$

В силу формулы Планшереля для любой функции $f \in W_{2}^{n}\left(\mathbb{R}^{1}\right)$ выполняется равенство

$$
\left\|\left.f\left|W_{2}^{n}\left(\mathbb{R}^{1}\right) \|^{2}=2 \pi \int_{-\infty}^{\infty}\right| \hat{f}(\xi)\right|^{2}\left(1+\xi^{2 n}\right) d \xi\right.
$$

а условие $f^{(s)}(0)=a_{s}, s \in I_{n}$, равносильно равенству $\int \xi^{s} \hat{f}(\xi) d \xi=(-i)^{s} a_{s}$. Поэтому $\hat{z}_{k}(\xi)$ является решением экстремальной задачи

$$
\int_{-\infty}^{\infty}|g(\xi)|^{2}\left(1+\xi^{2 n}\right) d \xi \rightarrow \min : \quad \int_{-\infty}^{\infty} \xi^{s} g(\xi) d \xi=(-i)^{s} \delta_{s, k}, s \in I_{n}
$$

Согласно общему принципу Лагранжа (см. например, [1, п. 1.5.4]), решение данной экстремальной задачи представимо в виде конечной линейной комбинации

$$
\hat{z}_{k}(\xi)=\sum_{j=0}^{n-1} c_{j, k} \frac{\xi^{j}}{1+\xi^{2 n}}
$$

Введем вектор $c^{(k)}$ с координатами $\left(c_{0, k}, \ldots, c_{n-1, k}\right)$ и матрицу $Q$ с элементами

$$
q_{j, k}:=\frac{1}{\pi} \int_{-\infty}^{+\infty} \frac{\xi^{j+k} d \xi}{1+\xi^{2 n}}=\frac{\varepsilon_{j, k}}{n \sin ((j+k+1) \theta)}, \quad \varepsilon_{j, k}:=\frac{1+(-1)^{j+k}}{2}, \theta:=\frac{\pi}{2 n} .
$$

Здесь использована хорошо известная формула для возникающего интеграла (см. [1, п. 2.4.4] и [5, п. 2.2.5.25]), а числитель отражает то очевидное обстоятельство, что этот интеграл равен 0 , если $j$ и $k$ - числа разной четности.

С помощью этой матрицы минимизируемый функционал (2.5) для функции (2.6) может быть записан как

$$
\int_{-\infty}^{\infty}\left|\hat{z}_{k}(\xi)\right|^{2}\left(1+\xi^{2 n}\right) d \xi=\sum_{j=0}^{n-1} \sum_{s=0}^{n-1} c_{j, k} \bar{c}_{k, s} \int_{-\infty}^{\infty} \frac{\xi^{j} \xi^{s} d \xi}{1+\xi^{2 n}}=2 \pi^{2}\left(Q c^{(k)}, c^{(k)}\right) .
$$


Если ввести вектор $\tilde{e}_{k}$ с координатами $(-i)^{k} \delta_{j, k}$, то ограничения в задаче (2.5) принимают вид

$$
(-i)^{s} \delta_{s, k}=\int_{-\infty}^{\infty} \xi^{s} \hat{z}_{k}(\xi) d \xi=\sum_{j=0}^{n-1} c_{j, k} \int_{-\infty}^{\infty} \frac{\xi^{j} \xi^{s} d \xi}{1+\xi^{2 n}} \Longleftrightarrow \tilde{e}_{k}=\pi Q c^{(k)}
$$

и, таким образом, $2 \pi^{2}\left(Q c^{(k)}, c^{(k)}\right)=2\left(Q^{-1} \tilde{e}_{k}, \tilde{e}_{k}\right) ;$ следовательно, числа $A_{n, k}^{2}$ суть диагональные элементы матрищъ $U$, обратной к матрице $Q$.

Шаг 3. Вывод явной формулы для диагональных элементов матрицы $U$. Нужные нам числа $u_{k, k}$ в силу особой структуры матрицы $Q$ могут быть вычислены конструктивно. Действительно, в силу (2.7) и формул Эйлера для всех $j, k \in I_{n}$ имеем

$$
\begin{gathered}
q_{j, k}=h_{j} \bar{h}_{k} \varepsilon_{j, k} v_{j, k}, \quad h_{j}:=e^{i j \theta}, \quad \theta:=\frac{\pi}{2 n} \\
v_{j, k}:=\frac{1}{\alpha_{j}+\bar{\alpha}_{k}}, \quad \alpha_{j}:=\frac{\exp (i(2 j+1) \theta)}{i n} .
\end{gathered}
$$

а отсюда явствует, что $Q=H \widetilde{V} \bar{H}$, где $H:=\operatorname{diag}\left(h_{0}, \ldots, h_{n-1}\right), \tilde{v}_{j, k}:=\varepsilon_{j, k} v_{j, k}$, а значит, $U=\bar{H} \widetilde{\Phi} H, \widetilde{\Phi}=\widetilde{V}^{-1}$, и, в частности $u_{k, k}=\widetilde{\varphi}_{k, k}$.

Переменные с четными и нечетными индексами разделяются, и для обращения матриц $V_{o}$ и $V_{e}$, образующих ненулевые блоки матрицы $\widetilde{V}$, соответствующие координатам с нечетными и четными индексами, мы применим утверждение (его доказательство, опирающееся на формулы для так называемых определителей Коши [6, §14], можно найти, например, в [7]), приводимое здесь в несколько большей общности.

ПРЕДЛОЖЕНИЕ 2. Для того чтобъ матрица $V$ с элементами $v_{j, k}:=$ $\left(\alpha_{j}+\beta_{k}\right)^{-1}$ была невырожденной, необходимо и достаточно, чтобы оба набора комплексных чисел $\left\{\alpha_{j}\right\}$ u $\left\{\beta_{k}\right\}$ состояли из попарно различных чисел; при выполнении этого условия диагональные элементы обратной матрицы $\Phi=V^{-1}$ равны

$$
\varphi_{k, k}=\left(\alpha_{k}+\beta_{k}\right) \prod_{r \neq k} \frac{\left(\alpha_{k}+\beta_{r}\right)\left(\beta_{k}+\alpha_{r}\right)}{\left(\alpha_{k}-\alpha_{r}\right)\left(\beta_{k}-\beta_{r}\right)}
$$

Соединяя приведенные рассуждения, мы заключаем из (2.9) с учетом (2.8), что для чисел $A_{n, k}$ справедлива явная формула

$$
A_{n, k}=\left(u_{k, k}^{(n)}\right)^{1 / 2}=(n \sin ((2 k+1) \theta))^{1 / 2} \prod_{r \in I_{n, k}} \frac{\sin (r+k+1) \theta}{\sin |r-k| \theta},
$$

где $I_{n, k}$ обозначает множество всех тех $r \in I_{n}$, для которых разность $r-k-$ четное число, не равное 0 , а произведения пустого множества чисел, возникающие в случаях $n=1, k=0 ; n=2, k=0, k=1 ; n=3, k=1$, принимаются, как обычно, равными 1.

Отметим, что именно в таком (промежуточном) виде выражение для констант $A_{n, k}$ было приведено в [8].

Шаг 4. Завершение доказательства теоремы: переход от (2.10) $к(1.3)$. 
Вводя обозначения (напомним, что теперь $\theta=\theta_{n}:=\pi / 2 n$ )

$$
a_{s}:=\sin s \theta, \quad P_{k}:=\prod_{s=1}^{k} a_{s}, \quad X_{m}=\prod_{0 \leqslant 2 s \leqslant m} a_{2 s+1}, \quad Y_{m}:=\prod_{0<2 s \leqslant m} a_{2 s},
$$

мы преобразуем выражение (2.10) к виду

$$
A_{n, k}=\sqrt{\frac{n}{a_{2 k+1}}} B_{n, k}, \quad B_{n, k}:=\frac{X_{k+n-1}}{X_{k-1} Y_{k} Y_{n-k-1}}=\frac{X_{2 n-2}}{P_{k} P_{n-k-1}} .
$$

Последний переход здесь проверяется непосредственным разбором четырех случаев четности $n, k$ с учетом равенств $a_{j}=a_{2 n-j}, j \in I_{n}$, имеющих место именно при $\theta=\theta_{n}$. Переходя в (2.12) от $k$ к $k+1$ и принимая во внимание тождества $\sin (n-j) \theta=\cos j \theta$, справедливые при выбранном $\theta$, получаем соотношение

$$
\frac{B_{n, k+1}}{B_{n, k}}=\frac{a_{n-k-1}}{a_{k+1}}=\frac{\sin (n-k-1) \theta}{\sin (k+1) \theta}=\operatorname{ctg}(k+1) \theta
$$

и, следовательно,

$$
A_{n, k}=\sqrt{\frac{n}{a_{2 k+1}}} B_{n, 0} \prod_{s=1}^{k} \operatorname{ctg} s \theta .
$$

Воспользуемся теперь известными формулами для произведений синусов (см., например, [5, пп. 6.1.2.5, 6.1.2.2]).

ПРЕДЛОЖЕНИЕ 3. При любом натуральном $n$ справедливы тождества

$$
\prod_{s=0}^{n-1} \sin \frac{(2 s+1) \pi}{2 n}=2^{(1-n) / 2}, \quad \prod_{s=0}^{n-1} \sin \frac{s \pi}{2 n}=2^{(1-n) / 2} \sqrt{n} .
$$

Первое произведение в (2.14) в соответствии с $(2.11)$ есть $X_{2 n-2}$, а второе равно $P_{n}$; таким образом, в силу $(2.12) B_{n, 0}=1 / \sqrt{n}$, а отсюда в соединении с (2.13) вытекает (1.3). Доказательство теоремы закончено.

\section{§3. Общие свойства констант $A_{n, k}$}

Из формулы (1.3) легко может быть выведено свойство симметрии, не очевидное с точки зрения исходного определения чисел $A_{n, k}$ (см. (1.2)).

СЛЕДСТВИЕ 1. Имеют место соотношения

$$
A_{n, n-k-1}=A_{n, k}, \quad n \in\{1,2, \ldots\}, k \in I_{n} .
$$

ДокАЗАтЕЛьство. Утверждение легко получается из равенства (2.12), равносильного $(2.10)$ и (1.3), с учетом тождества $\sin (2 k+1) \theta=\sin (2(n-k-1)+1) \theta$. Соотношение симметрии (3.1) вытекает также из факта симметричности матрицы $G_{n}$ относительно побочной диагонали (см. предложение 1$)$.

2 Функциональный анализ и его приложения, т. 38, вып. 3 
При $n \leqslant 6$ константы $A_{n, k}$, вычисляемые по формуле (1.3), могут быть точно выражены, исходя из натуральных чисел, посредством арифметических операций и извлечения квадратного корня (см. таблицу значений тригонометрических функций в [5, I.1.11]):

$$
\begin{gathered}
A_{1,0}=1, \quad A_{2,0}=A_{2,1}=\sqrt[4]{2}=1.1892 \ldots, \\
A_{3,0}=A_{3,2}=\sqrt{2}=1.4142 \ldots, \quad A_{3,1}=\sqrt{3}=1.7320 \ldots, \\
A_{4,0}=A_{4,3}=\sqrt[4]{4+2 \sqrt{2}}=1.6165 \ldots, \quad A_{4,1}=A_{4,2}=\sqrt[4]{20+14 \sqrt{2}}=2.5117 \ldots, \\
A_{5,0}=A_{5,4}=\sqrt{1+\sqrt{5}}=1.7989 \ldots, \\
A_{5,1}=A_{5,3}=\sqrt[4]{70+30 \sqrt{5}}=3.4217 \ldots, \quad A_{5,2}=2+\sqrt{5}=4.2360 \ldots, \\
A_{6,0}=A_{6,5}=\sqrt[4]{8+4 \sqrt{3}}=1.9656 \ldots, \quad A_{6,1}=A_{6,4}=\sqrt[4]{194+112 \sqrt{3}}=4.4381 \ldots, \\
A_{6,2}=A_{6,3}=\sqrt[4]{936+540 \sqrt{3}}=6.5771 \ldots
\end{gathered}
$$

Приведенная табличка наводит на предположение, что константы $A_{n, k}$ ведут себя так же регулярно, как, скажем, биномиальные коэффициенты $C_{n}^{k}$, которые для любого фиксированного $n$ монотонно возрастают по $k$ при $k<n / 2$. Это действительно имеет место, причем для отношения $A_{n, k+1} / A_{n, k}$ справедливы и более точные утверждения, требующиеся в некоторых вопросах.

СледСТвиЕ 2. (i) При увеличении $k$ отношение $A_{n, k+1} / A_{n, k}$ строго убъвает, тогдакак $(k+1) A_{n, k+1} / A_{n, k}$ строго возрастает;

(ii) $A_{n, k}$ строго возрастает при $k<m:=[(n-1) / 2]$, достигает при $k=m$ максимального значения и строго убывает при $k>m$;

$$
\begin{aligned}
& \text { (iii) } k+1<\frac{n}{4} \Longrightarrow \frac{2}{\pi \sqrt{3}} \frac{n}{k+1} \leqslant \frac{A_{n, k+1}}{A_{n, k}} \leqslant \frac{\sqrt{2}+1}{4} \frac{n}{k+1} \text {; } \\
& \text { (iv) } \frac{n}{4} \leqslant k+1<\frac{n}{2} \Longrightarrow 1 \leqslant \frac{A_{n, k+1}}{A_{n, k}} \leqslant \sqrt{2}+1,
\end{aligned}
$$

где константы $2 / \pi \sqrt{3},(\sqrt{2}+1) / 4,1, \sqrt{2}+1$ точные.

ДокАЗАтЕЛЬСтво. Непосредственно из (1.3) явствует, что при $k \in\{0, \ldots$, $n-2\}$

$$
\frac{A_{n, k+1}}{A_{n, k}}=\sqrt{\frac{\sin (2 k+1) \theta}{\sin (2 k+3) \theta}} \operatorname{ctg}(k+1) \theta,
$$

и, таким образом, утверждение (i) равносильно неравенству

$$
\frac{A_{n, k+1}}{A_{n, k}}\left(\frac{A_{n, k}}{A_{n, k-1}}\right)^{-1}=\sqrt{\frac{\sin ^{2}(2 k+1) \theta}{\sin (2 k-1) \theta \sin (2 k+3) \theta}} \frac{\operatorname{ctg}(k+1) \theta}{\operatorname{ctg} k \theta}<1
$$

$(k \in\{1,2, \ldots, n-2\})$, для доказательства которого рассмотрим функцию

$$
\mu(y, t):=\ln \sin 2(y+t)+\ln \sin 2(y-t)-2 \ln \sin 2 y+2 \ln \operatorname{tg}(y+t)-2 \ln \operatorname{tg}(y-t)
$$

в области $D:=\{y>t>0, y+t<\pi / 2\}$. Ее производная по $t$, равная

$$
\frac{\partial \mu(y, t)}{\partial t}=\frac{2(2+\cos 2(y+t))}{\sin 2(y+t)}+\frac{2(2-\cos 2(y-t))}{\sin 2(y-t)},
$$


положительна в $D$, и, учитывая, что $\mu(y, 0)=0$, мы заключаем, что в указанной области $\mu(y, t)>0$. Отсюда, потенцируя (3.4), получаем неравенство

$$
\frac{\sin 2(y+t) \sin 2(y-t)}{\sin ^{2} 2 y}>\frac{\operatorname{tg}^{2}(y-t)}{\operatorname{tg}^{2}(y+t)}, \quad y>t>0, y+t<\pi / 2,
$$

и, выбирая здесь $y:=(k+1 / 2) \theta, t:=\theta / 2$, приходим к (3.3); тем самым доказана первая половина части (i).

Вторая ее половина, выражающаяся неравенством

$$
\frac{(k+1) A_{n, k+1}}{A_{n, k}}\left(\frac{k A_{n, k}}{A_{n, k-1}}\right)^{-1}=\sqrt{\frac{\sin ^{2}(2 k+1) \theta}{\sin (2 k-1) \theta \sin (2 k+3) \theta}} \frac{\operatorname{ctg}(k+1) \theta}{\operatorname{ctg} k \theta} \frac{k+1}{k}>1,
$$

устанавливается аналогично и вытекает из отрицательности в области $D$ функции $\lambda(y, t):=\mu(y, t)+2 \ln (y-t)-2 \ln (y+t)$, что доказывается так же, как положительность $\mu(y, t)$.

Далее, согласно (3.2), при четных и нечетных $n$ имеем соответственно

$$
\frac{A_{2 m+2, m}}{A_{2 m+2, m+1}}=1, \quad \frac{A_{2 m+1, m-1}}{A_{2 m+1, m}}=\frac{A_{2 m+1, m}}{A_{2 m+1, m+1}}=\sqrt{\cos \frac{\theta}{2}} \operatorname{ctg}\left(\frac{\pi}{4}-\frac{\theta}{2}\right)>1,
$$

а отсюда в силу свойства (i) вытекает утверждение (ii).

Из доказанного свойства монотонности (i) мы получаем также (iii) и (iv), поскольку $A_{n, 1} / A_{n, 0}=2 n\left(1+\varepsilon_{n}\right) / \pi \sqrt{3}, \varepsilon_{n} \rightarrow+0, n \rightarrow \infty$ (cм. (3.2)), a $\operatorname{ctg} \pi / 8=$ $\sqrt{2}+1$. Доказательство следствия 2 закончено.

\section{§4. Асимптотическое поведение $\boldsymbol{A}_{n, k}$}

Для получения асимптотики величин (1.3) при $n \rightarrow \infty$ мы будем опираться на формулу суммирования Тейлора-Маклорена (см., например, [10, теорема 1, I]).

ПреДЛОЖЕНИЕ 4. Пусть $f(x)$ дваждъ непрерьвно дифберенцируема на отрезке $[a, b] ;$ тогда

$$
\begin{aligned}
\sum_{a<s \leqslant b} f(s) & =\int_{a}^{b} f(x) d x+\rho(b) f(b)-\rho(a) f(a)+\sigma(a) f^{\prime}(a)-\sigma(b) f^{\prime}(b) \\
& +\int_{a}^{b} \sigma(x) f^{\prime \prime}(x) d x, \quad \rho(x):=\frac{1}{2}-\{x\}, \sigma(x):=\int_{0}^{x} \rho(u) d u .
\end{aligned}
$$

Отсюда может быть выведена асимптотическая формула, представляющая и некоторый самостоятельный интерес.

Лемма 2. Пусть $k$ - натуралъное число, $\theta>0, \delta>0, y:=(k+1 / 2) \theta<$ $\pi / 2-\delta ;$ тогда

$$
\prod_{s=1}^{k} \operatorname{ctg} s \theta=\frac{1}{k ! \theta^{k}} \exp \left\{-\frac{Q(y)}{\theta}\right\}\left(1+\theta \varphi_{k}(\theta)\right)
$$

$2 \partial e$

$$
Q(y):=\int_{0}^{y} \ln \frac{\operatorname{tg} u}{u} d u, \quad\left|\varphi_{k}(\theta)\right|<c(\delta)
$$


ДоКАЗАТЕЛЬСтво. Запишем (4.1) при $a:=0, b:=k+1 / 2$ для функции

$$
f(x)=f(x, \theta):=g(\theta x), \quad g(x):=\ln \frac{\operatorname{tg} x}{x},
$$

и, учитывая, что $f(0)=\sigma(0)=0, \rho(0)=1 / 2, \rho(k+1 / 2)=0, \sigma(k+1 / 2)=1 / 8$, получим

$$
\begin{aligned}
T: & =\sum_{s=1}^{k} \ln \frac{\operatorname{tg} s \theta}{s \theta}=\sum_{s=1}^{k} f(s)=\int_{0}^{k+1 / 2} f(x) d x-\frac{1}{8} f^{\prime}\left(k+\frac{1}{2}\right)+\int_{0}^{k+1 / 2} \sigma(x) f^{\prime \prime}(x) d x \\
& =\frac{1}{\theta} Q(y)-\frac{\theta}{8} g_{1}(y)+\theta \int_{0}^{y} \sigma\left(\frac{u}{\theta}\right) g_{2}(u) d u, \quad g_{1}(u):=\frac{d g(u)}{d u}, g_{2}(u):=\frac{d^{2} g(u)}{d u^{2}} .
\end{aligned}
$$

Здесь в интегралах сделана линейная замена $u=x \theta$. Поскольку $0 \leqslant \sigma(x) \leqslant$ $1 / 8$ при всех $x$, а функции $g_{1}(u), g_{2}(u)$ ограничены на отрезке $[0, \pi / 2-\delta]$, модули двух последних слагаемых в (4.5) не превосходят $c(\delta) \theta$.

Из (4.4) и (1.3) вытекает соотношение

$$
\ln \prod_{s=1}^{k} \operatorname{ctg} s \theta=-\ln \left(k ! \theta^{k}\right)-T=-\ln \left(k ! \theta^{k}\right)-\frac{Q(y)}{\theta}-\frac{1}{2} g(y)+\theta \widetilde{\varphi}_{k}(\theta),
$$

которое равносильно (4.2). Доказательство леммы 2 закончено.

Выбирая в ней, как и ранее в $\S 2, \theta:=\pi / 2 n$, из формул (1.3) и (4.4) получаем

СледСТвИЕ 3. Пусть $n \rightarrow \infty$, а числа $k$ не превосходят $n(1-\delta), \delta>0$; тогда

$$
A_{n, k} \approx\left(\sin \frac{\pi(2 k+1)}{2 n}\right)^{-1 / 2}\left(\frac{2 n}{\pi}\right)^{k} \frac{1}{k !} \exp \left(-\frac{2 n}{\pi} Q\left(\frac{\pi(2 k+1)}{4 n}\right)\right),
$$

где символ $\approx$, как обычно, означает, что отночение правой части к левой стремится $\kappa 1$ равномерно относительно $k$, лежащих в указанных промежутках.

Отметим, что в силу симметрии нам вполне достаточно ограничиться рассмотрением значений $k<n / 2$, что соответствует выбору $\delta=1 / 2$.

В ряде случаев данная общая формула принимает более простой вид.

СлеДСТВИЕ 4 . Пусть $n \rightarrow \infty$, а отношение $k^{3} / n^{2}$ ограничено; тогда

$$
A_{n, k} \approx\left(\frac{2 n}{\pi}\right)^{k+1 / 2} \frac{1}{k ! \sqrt{2 k+1}} \exp \left(-\frac{\pi^{2} k^{3}}{36 n^{2}}\right) .
$$

ДокАзАТЕЛЬСтво. Условие следствия подразумевает, что $k / n \rightarrow 0$, а значит, $\sin (\pi(2 k+1) / 2 n) \approx \pi(2 k+1) / 2 n$. Из (4.3) вытекает, что $Q(y) \approx y^{3} / 9, y:=$ $\pi(2 k+1) / 4 n \rightarrow 0$, а это в соединении с (4.6) дает (4.7).

Отметим, что в случае, когда $k=o\left(n^{2 / 3}\right)$, в частности, для любого фиксированного $k$, последний сомножитель в (4.7) можно отбросить, поскольку он стремится к 1 .

СлеДСтвие 5 . Пусть $k \rightarrow \infty,(n-k) \rightarrow \infty, \alpha:=(k+1 / 2) / n ;$ тогда

$$
A_{n, k} \approx \frac{\exp (n K(\alpha))}{2 \sqrt{n \sin (\pi \alpha)}}, \quad K(\alpha):=\frac{2}{\pi} \int_{0}^{\pi \alpha / 2} \ln (\operatorname{ctg} x) d x .
$$


ДокАЗАтЕЛьСтво. Отметим сначала, что функция $K(\alpha)$ положительна, возрастает на $(0,1 / 2)$, достигает наибольшего значения $K_{0}=K(1 / 2)=0.583 \ldots=$ $\ln 1.79 \ldots$ и удовлетворяет (как и знаменатель в формуле (4.8) для $A_{n, k}$ ) условию симметрии $K(1-\alpha)=K(\alpha)$, причем переход от $\alpha$ к $1-\alpha$ в точности соответствует переходу от индекса $k \mathrm{k} n-k-1$. Поэтому мы можем (и будем) далее считать, что $\alpha \leqslant 1 / 2$, т. е. $k \leqslant(n-1) / 2$.

Из сопоставления (4.8) и (4.3) явствует, что

$$
K(\alpha)=\frac{2}{\pi}\left(-Q\left(\frac{\pi \alpha}{2}\right)-\int_{0}^{\pi \alpha / 2} \ln x d x\right)=\frac{2}{\pi} Q\left(\frac{\pi \alpha}{2}\right)+\alpha\left(1+\ln \frac{2}{\pi \alpha}\right)
$$

а отсюда, с учетом равенства $\alpha n=k+1 / 2$, вытекает соотношение

$$
\exp (n K(\alpha))=\exp \left(-\frac{2 n}{\pi} Q\left(\frac{\pi(2 k+1)}{4 n}\right)\right)\left(\frac{4 n e}{\pi(2 k+1)}\right)^{k+1 / 2} .
$$

Далее, поскольку $k \rightarrow \infty$, из формулы Стирлинга следует, что

$$
\left(\frac{4 n e}{\pi(2 k+1)}\right)^{k+1 / 2}=\frac{\sqrt{4 n e}}{\sqrt{\pi(2 k+1)}}\left(\frac{e}{k}\right)^{k}\left(\frac{2 n}{\pi}\right)^{k}\left(\frac{2 k}{2 k+1}\right)^{k} \approx \frac{2 \sqrt{n}}{k !}\left(\frac{2 n}{\pi}\right)^{k} .
$$

Соединяя (4.11), (4.10) и (4.6), получаем (4.8); следствие 5 доказано.

ЗАмЕЧАНИЕ 1. Из (4.8) вытекает асимптотика максимального (при заданном $n)$ из чисел $A_{n, k}$, которое в силу свойства регулярности (следствие 2 ) есть, разумеется, $A_{n,[n-1 / 2]}$, а именно: $\max _{k} A_{n, k} \approx e^{n K_{0}} / 2 \sqrt{n}$; более грубый результат $\max _{k} \ln A_{n, k} \approx n K_{0}$ был установлен ранее в [4].

ЗАмЕчАниЕ 2. Переходя в (4.8) к логарифмам, устремляя $\alpha$ к 0 и замечая, что при этом первое слагаемое в конце цепочки (4.9) бесконечно мало относительно второго, получаем утверждение: если $n, k, n / k \rightarrow \infty, \operatorname{mo} \ln A_{n, k} \approx$ $k \ln (n / k)$.

ЗАМЕчАНИЕ 3. Используя известный прием [1, п. 2.4.3], включающий растяжения $f(x) \rightarrow f(t x), t>0$, мы можем перейти от (1.2) к равносильным неравенствам типа Ландау-Колмогорова с точными константами в мультипликативной форме:

$$
\begin{gathered}
\left\|f^{(k)}\right\|_{L_{\infty}\left(\mathbb{R}_{+}^{1}\right)} \leqslant K_{n, k}\|f\|_{L_{2}\left(\mathbb{R}_{+}^{1}\right)}^{\beta}\left\|f^{(n)}\right\|_{L_{2}\left(\mathbb{R}_{+}^{1}\right)}^{\alpha}, \\
K_{n, k}:=A_{n, k} \alpha^{-\alpha / 2} \beta^{-\beta / 2}, \quad \alpha=\alpha_{n, k}:=\frac{2 k+1}{2 n}, \quad \beta=\beta_{n, k}:=1-\alpha_{n, k} .
\end{gathered}
$$

Автор выражает глубокую благодарность В. М. Тихомирову, В. И. Буренкову и Л. Д. Кудрявцеву за внимание к работе, а также С. М. Никольскому, Г. Г. Магарил-Ильяеву и С. И. Похожаеву за ряд ценных замечаний.

\section{ЛитЕРАТУРА}

1. Тихомиров В. М. Некоторые вопросы теории приближений. Изд-во МГУ, М., 1976.

2. Габушин B. Н. О наилучшем приближении оператора дифференцирования на полуоси. Матем. заметки, 6, №5, 573-582 (1969).

3. Тайков Л. В. Неравенства колмогоровского типа и наилучшие формулы численного дифференцирования. Матем. заметки, 4, №2, 233-238 (1968). 
4. Калябин Г. А. Наилучшие операторы продолжения для соболевских пространств на полупрямой. Функц. анализ и его прил., 36, вып. 2, 28-37 (2002).

5. Прудников А. П., Брычков Ю. А., Маричев О. И. Интегралы и ряды. Наука, М., 1981.

6. Ахиезер Н. И. Лекции по теории аппроксимации. Наука, М., 1965.

7. Kalyabin G. A. On extrapolation with minimal norms in Bernstein classes. Proc. Razmadze Math. Inst., 119, 85-92 (1999).

8. Калябин Г. А. О точных константах в неравенствах Колмогорова для пространств Соболева $W_{2}^{n}\left(\mathbb{R}_{+}\right)$. Докл. РАН, 388, № 2, 159-161 (2003).

9. Калябин $\Gamma$. А. Асимптотика наименьших собственных значений матриц типа Гильберта. Функц. анализ и его прил., 35, вып. 1, 80-84 (2001).

10. Карачуба А. А. Основы аналитической теории чисел. Наука, М., 1983.

Институт систем обработки изображений РАН

Самарская гуманитарная академия

Поступило в редакцию

email: kalyabin@mb.ssau.ru, kalyabin@lycos.com

16 июня 2003 г. 